Biosensors and Bioelectronics, Volume 22, Issue 13, 15 September 2006, pages 381-387

\title{
The Influence of Initiator and Different Polymerisation Conditions on the Performance of Molecularly Imprinted Polymers
}

Irene Mijangos, Fernando Navarro-Villoslada, Antonio Guerreiro, Elena Piletska, Iva Chianella, Kal Karim, Anthony Turner, Sergey Piletsky

Contribution from the Institute of BioScience and Technology, Cranfield University, Silsoe, Bedfordshire, MK45 4DT, UK

\begin{abstract}
A set of polymers was imprinted with (-)-ephedrine using two different initiators. A chemometrics approach was used to optimise experiments aimed at an analysis of the interplay of parameters such as polymerisation time, temperature and percentage of initiator and their role in the morphology and performance of materials synthesized for enantio-separation. The results presented demonstrate the importance of keeping the right balance between various polymerisation conditions. It is shown that enhancing one single parameter such as polymer rigidity does not necessarily improve polymer performance. In general it could be concluded that MIPs should be synthesized over a long period of time using low concentration of initiator and low temperature. The best selectivity was achieved for polymers prepared by photoinitiation with 2,2-dimethoxy-2-phenylacetophenone as initiator.
\end{abstract}


"To whom correspondence should be addressed. Tel: +44 (0)1525 863584, fax: +44 (0)1525863533, E-mail: $\underline{\text { S.Piletsky@cranfield.ac.uk }}$

\section{Introduction}

The relatively slow progress in commercialization of molecularly imprinted polymers (MIPs) is related entirely to the inadequate performance of this class of synthetic receptors in practical applications. Basically the polymers have not provided sufficient competitive advantage over other techniques and materials used in analytical chemistry and in separation to warrant their use.. The main reason for this lies in a poor understanding of the factors which govern the recognition properties of MIPs. The realization of this limitation recently led to the development of a range of projects where researchers re-visited the fundamentals of design and performance of imprinted polymers. ${ }^{1,2}$ This work involved comprehensive analysis of the type and concentration of the monomers used in polymer preparation, ${ }^{3,4}$ polymerisation temperature, ${ }^{5,6}$ pressure, $^{7,8}$ solvent, ${ }^{9,10}$ polymerisation time and polymer swelling properties. $^{11}$

Here we focus on studying another factor critically important for MIP design,initiators and their role in forming high performance MIPs. We have used chemometric tools to link together several parameters such as concentration of initiator and polymerisation time for two different initiator molecules, 1,1'-azobis(cyclohexane-1-carbonitrile) (ACC) and 2,2-dimethoxy-2-phenylacetophenone (DMPA). It was expected that the polymers formed with higher percentages of initiator would be more rigid, which would ensure a more defined shape of imprinting cavities and higher specificity of MIPs. On other hand, the lower amounts of initiator would decrease the temperature reached in the polymerisation mixture, which is crucial for the formation of good quality imprinting cavities. Thus we intended to find a balance between the rigidity of 
imprinting cavities versus the quality of the imprinting cavities, which would be necessary for the polymer to have good recognition properties. To make the study generic, we chose two different initiators which have slightly different initiation mechanisms.

Many organic molecules that are stable can undergo bond dissociation when exposed to a certain amount of energy (e.g. the thermal energy of ultraviolet light), and can be used as initiators of radical polymerisation. ${ }^{12}$ The appropriate use of the initiator will control the degree of the exothermic process of polymerisation. As each initiator has its own specific decomposition rate at a given temperature, the concentration and the curing conditions will be of great importance as well. ${ }^{13}$ Consequently, each initiator will decompose in different ways.

Azoinitiators ( $\mathrm{R}-\mathrm{N}=\mathrm{N}-\mathrm{R})$ normally have tertiary $\mathrm{R}$ groups that are able to stabilize the incipient radical. ${ }^{14}$ Figure 1 shows the mechanism of decomposition of the initiator 1,1-azobis(cyclohexane-1-carbonitrile) used in our experiments.

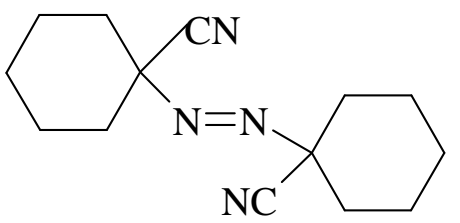

1',1-azobis(cyclohexanecarbonitrile)
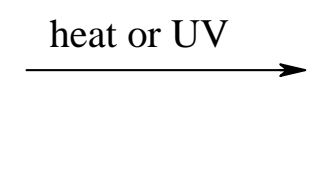

free radical

Figure 1. Mechanism of decomposition for 1',1-azobis(cyclohexanecarbonitrile), based on the mechanism of decomposition of AIBN. ${ }^{12}$ 
Acetophenone derivatives generate radicals by a very fast photochemical cleavage reaction followed by a second fragmentation, process that is not quenched by oxygen. ${ }^{14}$<smiles>CO[C+](OC)c1ccccc1</smiles><smiles>COC(=O)c1ccccc1</smiles>

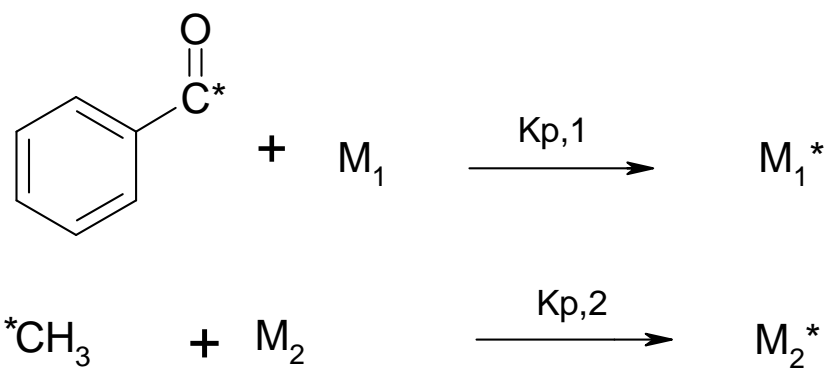

Figure 2. Mechanism of decomposition of the DMPA. ${ }^{15}$

In the scheme, $\mathrm{k}_{\mathrm{d}, \mathrm{i}}$ is the rate constant for the decomposition of the initiator into the benzoyl radical and the benzoyl ketal radical, $\mathrm{k}_{\mathrm{d}, \mathrm{r}, 1}$ is the rate constant for the decomposition of the benzoyl ketal radical to form methyl benzoate and methyl radical, $\mathrm{k}_{\mathrm{p}, 1}$ is the rate constant for the benzoyl radical-monomer reaction and $\mathrm{k}_{\mathrm{p}, 2}$ is the rate constant for the methyl radical-monomer reaction.

These two initiators have been used to prepare a set of hydroxyethyl methacrylate (HEMA) based polymers which have been imprinted with (-)-ephedrine using two 
different initiation protocols: thermo- and UV polymerisation. This model system has been introduced previously for studying the effects of polymerisation temperature and pressure, time of polymerisation and polymer swelling. ${ }^{6,8,11}$ The synthesized materials were studied by HPLC and their morphology, swelling data and performance compared. Chemometrics were used to link together several parameters, such as concentration of the initiator and time of the polymerisation, with the performance of synthesized polymers.

\section{Results and Discussion}

To analyze the effect of the percentage of initiator on the MIP's affinity and specificity, different sets of (-)-ephedrine imprinted polymers were synthesized and packed in chromatographic columns and tested by HPLC in chloroform with hexamethylenediamine (HMDA) for their ability to interact with (+)- and (-)ephedrine under similar conditions. In the first set of experiments (Table 1), the polymers were initiated by thermo-initiation at $80{ }^{\circ} \mathrm{C}$, using 1,1 '-azobis(cyclohexane1-carbonitrile) (ACC) as an initiator. In the second set of experiment (Table 2) the polymers were initiated under a UV lamp $\left(0.016 \mathrm{~W} / \mathrm{cm}^{2}\right)$ on ice, using $1,1^{\prime}$ azobis(cyclohexane-1-carbonitrile) (ACC) as initiator. In the third set of experiments

(Table 3) the polymers were synthesized under a UV lamp $\left(0.016 \mathrm{~W} / \mathrm{cm}^{2}\right)$ on ice, using 2,2-dimethoxy-2-phenylacetophenone (DMPA). 
Table 1. Polymers synthesized and studied using ACC as initiator. The reaction was initiated thermally at $80{ }^{\circ} \mathrm{C}$.

\begin{tabular}{|c|c|c|}
\hline Polymer & Percentages of ACC (\%) & Time of polymerisation (hours) \\
\hline MIP1 & $1 \%$ & 0.5 \\
\hline MIP2 & $1 \%$ & 3 \\
\hline MIP3 & $1 \%$ & 72 \\
\hline MIP4 & $1 \%$ & 106 \\
\hline MIP5 & $1 \%$ & 192 \\
\hline MIP6 & $1 \%$ & 0.5 \\
\hline MIP7 & $3 \%$ & 3 \\
\hline MIP8 & $3 \%$ & 192 \\
\hline MIP9 & $3 \%$ & 0.5 \\
\hline MIP10 & $5 \%$ & 192 \\
\hline MIP11 & $5 \%$ & \\
\hline
\end{tabular}

Table 2. Polymers synthesized and studied using ACC as initiator. The reaction was initiated by UV irradiation at $0{ }^{0} \mathrm{C}$.

\begin{tabular}{|c|c|c|}
\hline Polymer & Percentages of ACC (\%) & Time of polymerisation (hours) \\
\hline MIP12 & $1 \%$ & 0.5 \\
\hline MIP13 & $1 \%$ & 6 \\
\hline MIP14 & $1 \%$ & 12 \\
\hline MIP15 & $3 \%$ & 0.5 \\
\hline MIP16 & $3 \%$ & 6 \\
\hline MIP17 & $3 \%$ & 12 \\
\hline
\end{tabular}




\begin{tabular}{|c|c|c|}
\hline MIP18 & $5 \%$ & 0.5 \\
\hline MIP19 & $5 \%$ & 6 \\
\hline MIP20 & $5 \%$ & 12 \\
\hline
\end{tabular}

Table 3. Polymers synthesized and studied using DMPA as initiator. The reaction was initiated by UV irradiation at $0{ }^{0} \mathrm{C}$.

\begin{tabular}{|c|c|c|}
\hline Polymer & Percentages of DMPA (\%) & Time of polymerisation (hours) \\
\hline MIP21 & $1 \%$ & 0.5 \\
\hline MIP22 & $1 \%$ & 2 \\
\hline MIP23 & $1 \%$ & 3 \\
\hline MIP24 & $1 \%$ & 12 \\
\hline MIP25 & $1 \%$ & 3 \\
\hline MIP26 & $3 \%$ & 6 \\
\hline MIP27 & $3 \%$ & 0.5 \\
\hline MIP28 & $5 \%$ & 3 \\
\hline MIP29 & $5 \%$ & 10 \\
\hline MIP30 & $5 \%$ & \\
\hline
\end{tabular}

For each set of experiments, MIPs were synthesized a) for different periods of time, varying from a minimum of 1 hour to a maximum of 192 hours (for the thermoinitiated polymers), and a minimum of half an hour and a maximum of 12 hours (for the UV-initiated polymers), and b) with different percentages of initiator i.e. 1, 3 and $5 \%$ of initiator. Concentrations of initiator below $1 \%$ were, in most cases, insufficient for the creation of rigid polymers.

The result of this evaluation has been expressed in terms of percentage of initiator and time dependence of separation factors for ephedrine enantiomers. The intention was to link together the variation in polymerisation conditions with observed changes in 
polymer selectivity (expressed as separation factor). Alternatively we could have focused on studying variations in polymer affinity. This would, however, require a multitude of tests of blank polymers, which would dramatically increase the required volume of analytical work and would complicate the data analysis. Due to this, the model system we chose was focused specifically on studying enantio-separation using MIPs. We have noted however, that in all these experiments a direct correlation was observed between polymer affinity and selectivity. Thus the polymers which demonstrated higher separation factors possessed higher affinity (capacity factors) for both enantiomers.

The results of testing the synthesized polymers are presented in Figures 3-5. Several important conclusions can be drawn from this study. Firstly, it can be seen clearly that the thermo-initiation produces polymers with inferior properties as compared with photo-initiation. This is not surprising and as explained earlier, it results from the negative effect which increased temperature has on the complex formation between template and functional monomers. ${ }^{6}$ 


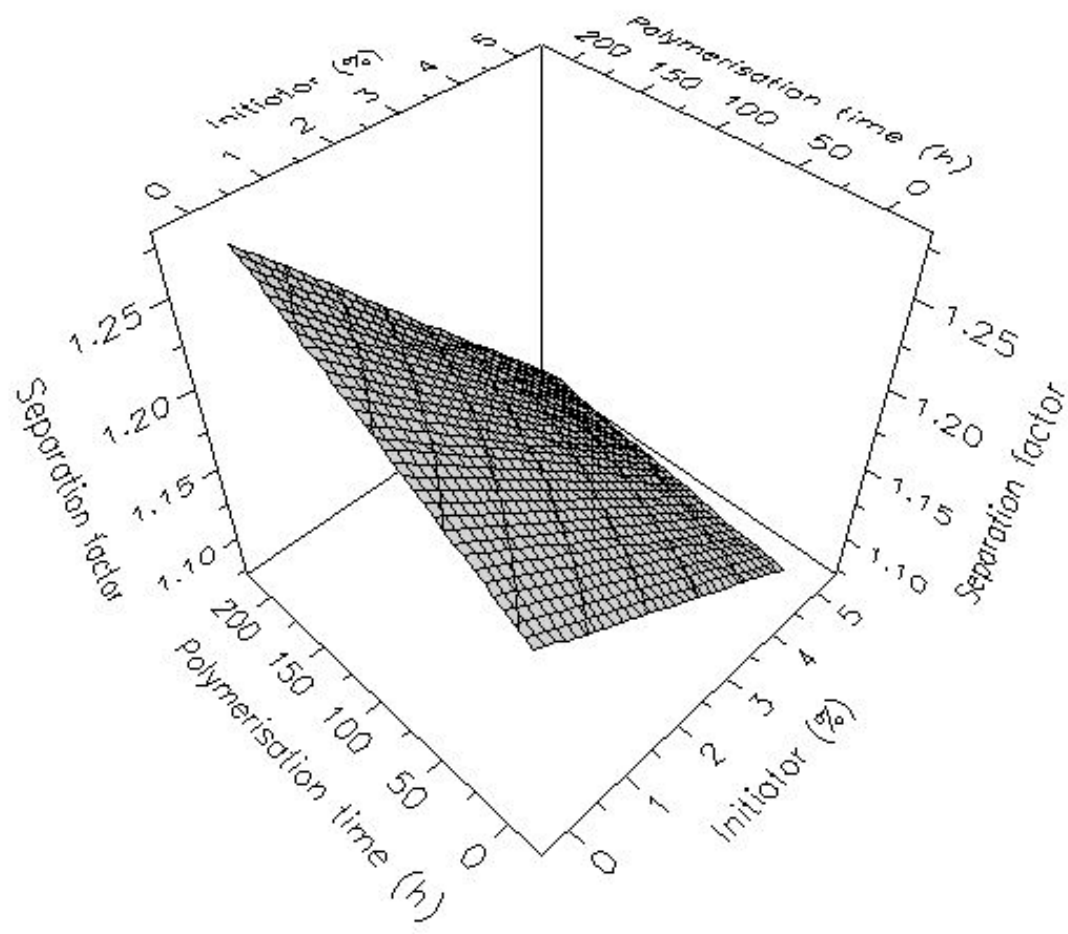

Figure 3. Influence of the polymerisation time and ACC percentage on separation factor $(\alpha)$ for polymers synthesized in the oil bath at $80{ }^{\circ} \mathrm{C}$.

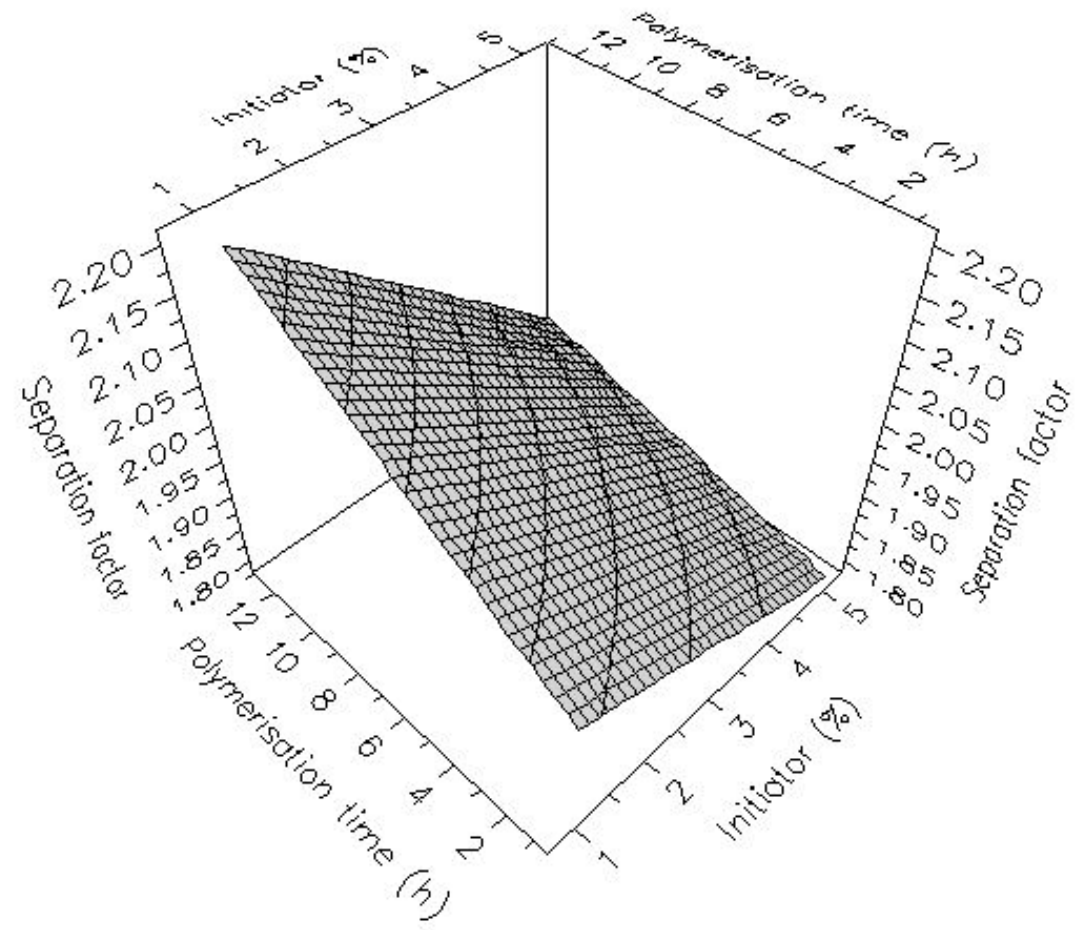

Figure 4. Influence of the polymerisation time and ACC percentage on separation factor $(\alpha)$ for polymers synthesized under the UV source. 


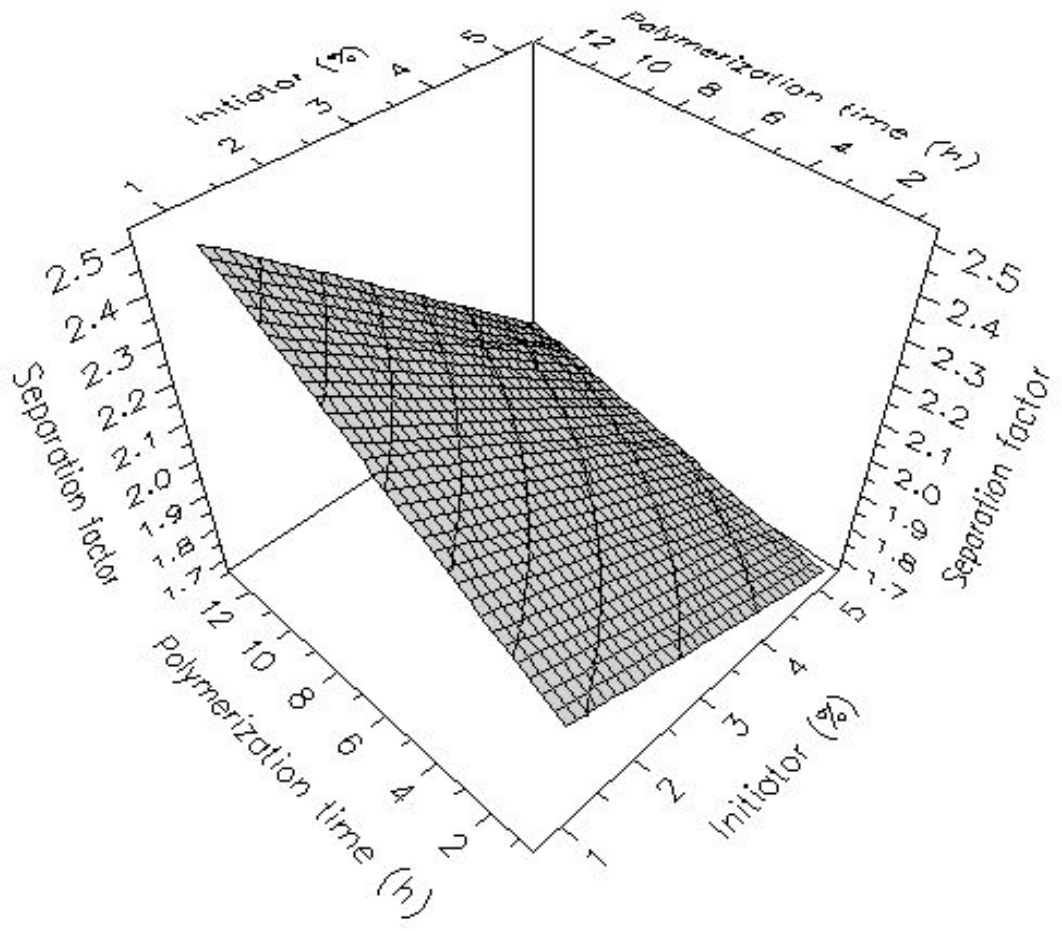

Figure 5. Influence of the polymerisation time and DMPA percentage on separation factor $(\alpha)$ for polymers synthesized under the UV source.

Secondly, it can be concluded that on average large concentrations of initiator hve detrimental effects on the recognition properties of synthesized polymers. It could be possible that the monomer molecules interfere with the complexation process between monomers and template. A detailed modeling study, performed in our laboratory, where we looked at the energetics of monomer-template, initiator-template and initiator-monomer interactions, indicated that this is unlikely. A second explanation includes the role of the heat generated during the polymerisation reaction. Thus we assume that large concentrations of initiator added to monomer mixture should increase the polymerisation rate and increase the heat of reaction. ${ }^{16}$ The increased temperature would disrupt the complex formed between template and monomers and reduce the affinity and selectivity of MIPs. 
The temperature profile of polymerisation reactions for all the processes studied is shown in Figures 6-8. The temperature of the polymerisation reaction was monitored using a thermocouple placed in the middle of the polymerisation vessel. The monitoring lasted for the first hour,the period which, for this model system, determines the quality of imprints formed. ${ }^{11}$

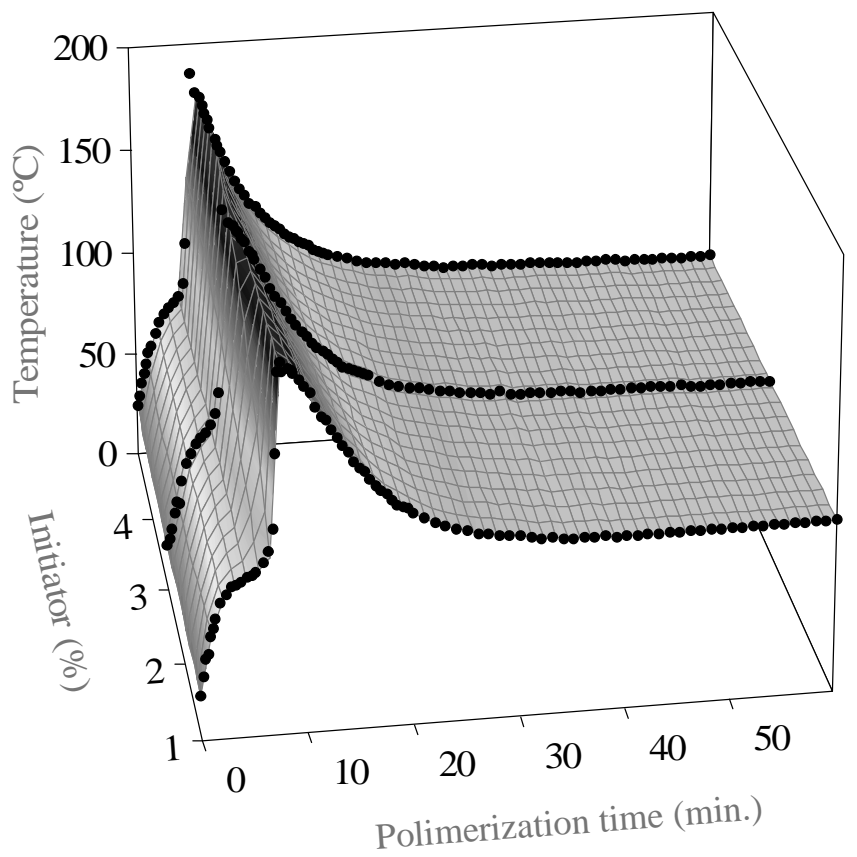

Figure 6. The temperature profile of the polymerisation reaction with different percentages of ACC for the polymers synthesized in an oil bath at $80{ }^{\circ} \mathrm{C}$. 


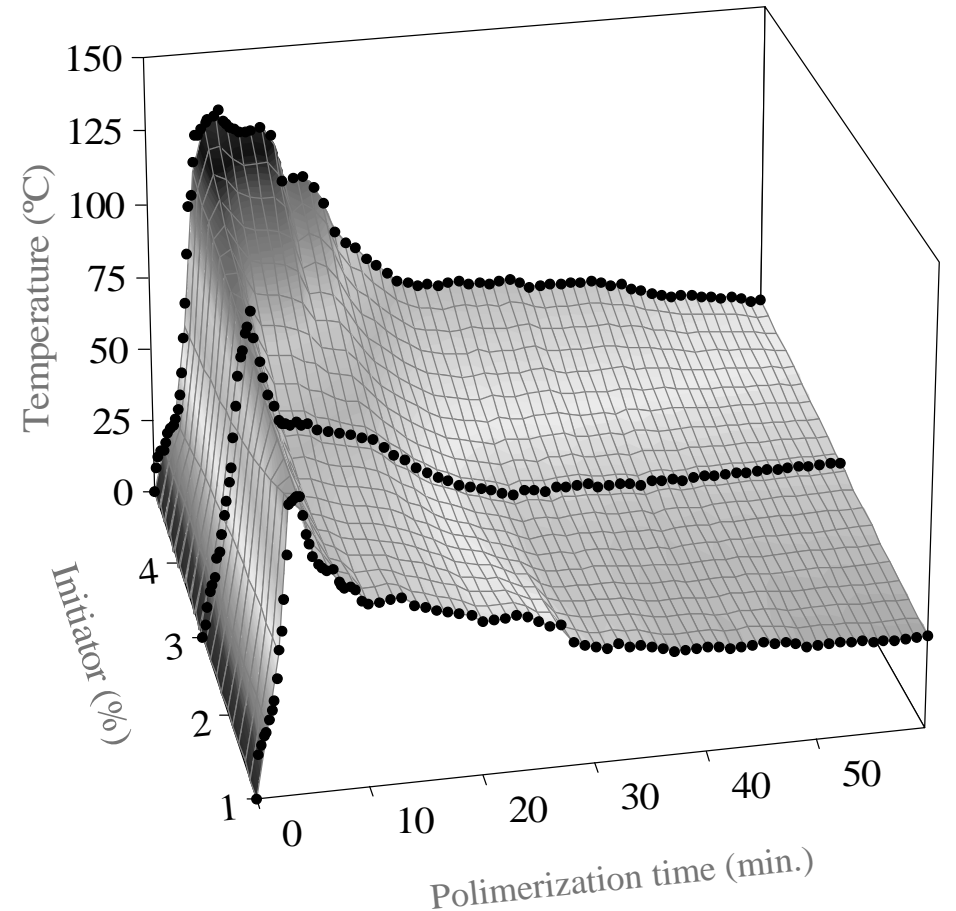

Figure 7. The temperature profile of the polymerisation reactions with different percentages of ACC for the polymers synthesized under the UV source.

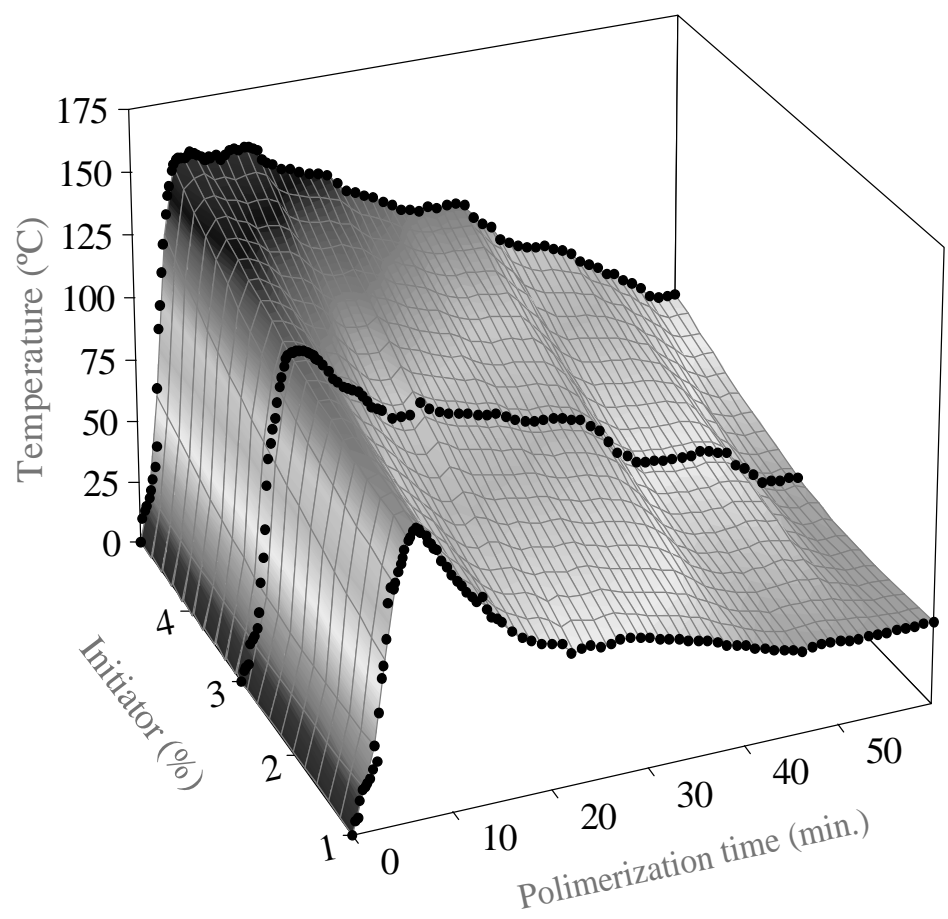

Figure 8. The temperature profile of the polymerisation reactions with different percentages of DMPA for the polymers synthesized under the UV source. 
As expected, larger concentrations of used initiator generated more heat during the polymerisation. More heat was also generated during the thermo-initiated than with the UV initiated reaction. By comparing these results with those showing polymer performance (Figures 2-5) it is possible to conclude that the temperature released during polymerisation is at least one of the major factors, if not the major factor, which determines the variations in polymer performance observed in our experiments.

What was unexpected however, is the observation that there seemed to be no limit to the tendency that the lower quantity of initiator produced polymer with superior recognition abilities. It has been shown before that polymer rigidity is critically important for enantio-separation. ${ }^{11}$ It would be reasonable to assume that lower concentration of initiator would lead to larger amounts of non-polymerizable double bonds remaining in the polymer and thus produce less rigid polymers. The higher concentration of initiator would lead to the formation of a larger number of free radicals and a larger number of growing nuclei and globules which would, however, have smaller size. Polymer composed of smaller globules will have a larger number of smaller pores and a larger surface area. This is consistent with an analysis of nitrogen adsorption isotherms (BET) performed for range of MIPs prepared by thermoinitiation (Table 4). In general, MIPs prepared using larger concentrations of initiators possessed a larger surface area. The total pore volume and average pore diameter varied however, perhaps due to the porogenic ability of the solvent vapors released during the highly exothermic reaction. ${ }^{8}$ 
Table 4. Influence of polymerisation time on the polymer morphology. All MIPs were polymerised for 7 days in an oil bath at $80{ }^{\circ} \mathrm{C}$ using ACC.

\begin{tabular}{cccc}
\hline $\begin{array}{c}\text { ACC } \\
\%\end{array}$ & $\begin{array}{c}\text { BET Surface area, } \\
\mathrm{m}^{2} \mathrm{~g}^{-1}\end{array}$ & $\begin{array}{c}\text { Total pore volume, } \\
\mathrm{cc} \mathrm{g}^{-1}\end{array}$ & $\begin{array}{c}\text { Aver. pore diameter, } \\
\mathrm{nm}\end{array}$ \\
\hline 1 & 56.94 & 0.155 & 5.5 \\
\hline 3 & 57.65 & 0.31 & 10 \\
\hline 5 & 72.59 & 0.302 & 8.3 \\
\hline
\end{tabular}

The influence of the polymerisation conditions on the rigidity of MIPs prepared using thermo- and UV-initiation has been studied as described earlier. ${ }^{11}$ As expected higher concentrations of initiator and prolonged polymerisation time decreased polymer swelling (Figure 9). Very similar results were obtained for all polymeric systems under investigation.

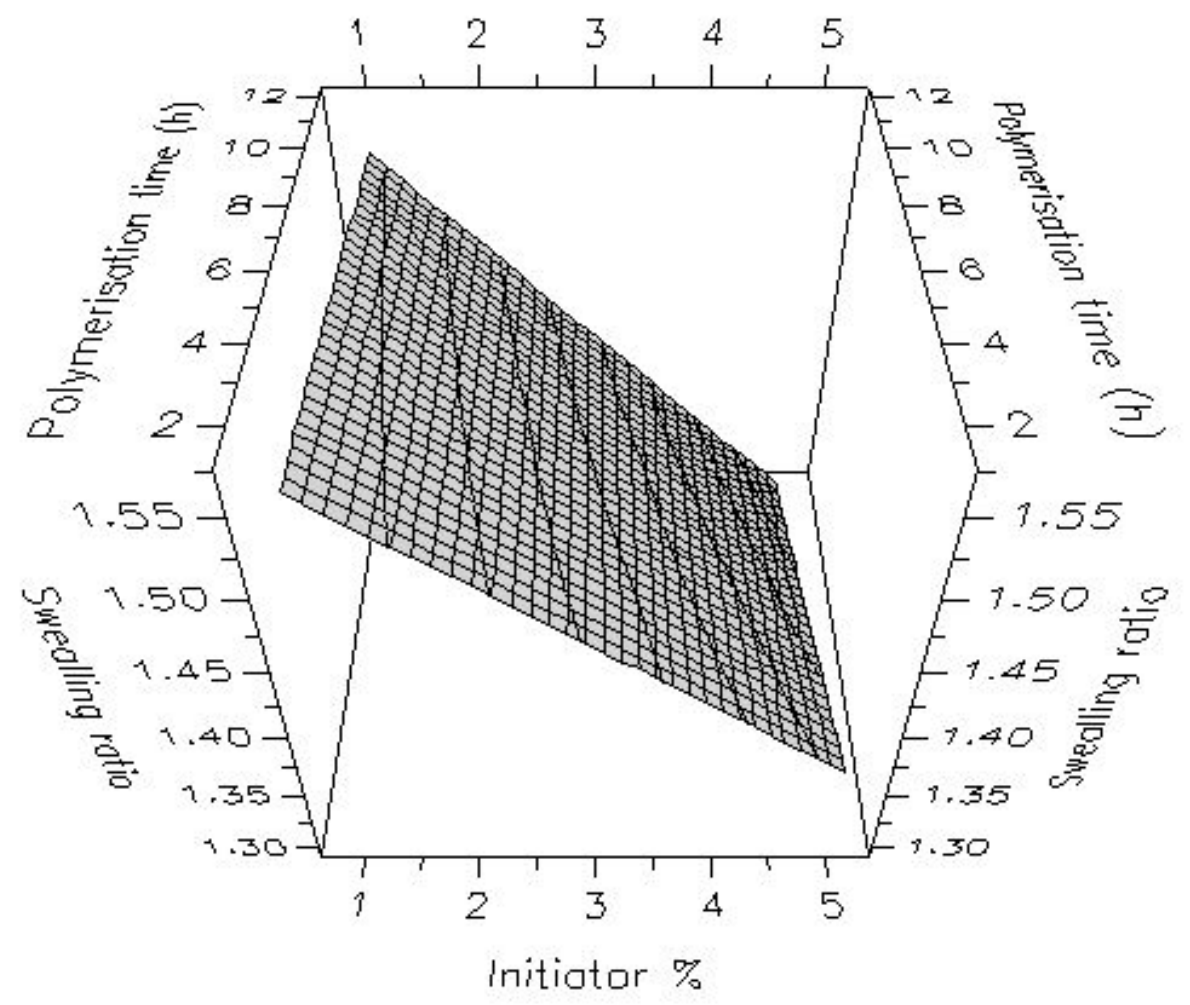

Figure 9. Influence of the polymerisation time and ACC percentage on swelling ratio for polymers synthesized under the UV source. 
A correlation does not exist, however, between polymer selectivity and swelling. The most rigid materials prepared using high concentrations of initiator and long polymerisation times, were less selective than less rigid MIPs polymerised using low concentrations of initiator. Apparently, high temperatures produced during polymerisation with large quantities of initiator overweighed (in negative terms) the positive impact of polymer rigidity. The general conclusion made from this work is that in order to achieve the best performance in enantio-separation (and possibly other types of applications), MIPs should be synthesized over a long period of time using low concentrations of initiator and low temperatures.

\section{Conclusion}

A chemometrics approach has been used to optimise experiments aimed at analysis of the interplay of parameters such as polymerisation time, temperature and percentage of initiator and their role in the morphology and the performance of synthesized materials. The present results demonstrate the importance of keeping the right balance between various polymerisation conditions. Thus the high polymer rigidity achieved with large concentrations of initiator had a negative impact on polymer performance. At the same time, at low concentrations of initiator, the higher rigidity achieved upon longer polymerisation time had a beneficial effect. This is due to the fact that the high temperatures reached with high concentrations of initiator worsened the quality of imprints formed. The lower quantities of initiator the lower was the temperature generated during polymerisation and the better were the recognition properties of MIPs. In general, it can be concluded that MIPs should be synthesized for long period 
of time using low concentrations of initiator and low temperatures. The best selectivity was achieved for polymers prepared by photoinitiation with 2,2dimethoxy-2-phenylacetophenone as initiator. As mentioned before, radical generation for acetophenone derivatives is a process that is not quenched by oxygen and this would be beneficial for many practical applications including sensors. ${ }^{17}$

\section{Experimental Section}

Chemicals. 1R,2S-ephedrine ((-)-ephedrine) and 2R,1S ephedrine ((+)-ephedrine) were supplied by Chemical Development, GlaxoSmithKline R\&D, UK. Ethylene glycol dimethacrylate (EGDMA), 2-hydroxyethyl methacrylate (HEM), 1,1'-azobis (cyclohexanecarbonitrile), 2,2-dimetoxy-2-phenylacetophenone, hexamethylenediamine (HMDA) and chloroform were purchased from Aldrich (UK). Diethyldithiocarbamic acid benzyl ester was supplied by TCI Europe nv. All chemicals and solvents were analytical or HPLC grade and were used without further purification.

Chemometic optimisation of the experiment. The polymer design and modeling were carried out using MODDE 6.0 software (Umetrics, Sweden) with two factors in the experimental design: (i) the amount of initiator and (ii) the polymerisation time. The influence of these two factors on the separation factor $(\alpha)$ was modelled by multivariate analysis. A two-level full factorial design was established for each initiator and polymerisation method. In addition, an experiment carried out at the centre of the experimental region was added to determine whether there is a linear or non-linear relationship between the factors and the response (separation factor).

The separation factor values obtained with the experimental design were related to the experimental parameters by an empirical model obtained using a partial least squares 
(PLS) regression method. The degree of fit and the prediction ability of the models were taken into account to select the model complexity, i.e., the number of regression coefficients included in the model. The interpretation on the models was assessed by the relative sizes and signs of the coefficients that indicated those that will contribute more strongly to the modelling of the response. ${ }^{4}$

Preparation of molecularly imprinted polymers. Several sets of imprinted polymers were synthesized with the different initiation methods and different initiators. To a solution of (+)-ephedrine (1.21 mmol, $0.2 \mathrm{~g})$ in chloroform $(8.82 \mathrm{~g})$ was added 2-hydroxyethyl methacrylate (12.1 mmol, $1.57 \mathrm{~g})$, ethylene glycol dimethacrylate (35.9 mmol, $7.1 \mathrm{~g}$ ) and 1,1'-azobis (cyclohexanecarbonitrile) or 2,2dimetoxy-2-phenylacetophenone $(0.18,0.54$ or $0.9 \mathrm{~g})$. The monomer mixture was placed in a $50 \mathrm{ml}$ glass tube and purged with nitrogen for 5 minutes. The polymerisation was initiated using (i) a fibre optic light source with a $0.016 \mathrm{~W} / \mathrm{cm}^{2}$ intensity CERMAX® Xenon Arc Lamp (PerkinElmer Optoelectronics, Inc., USA) with the mixture in an ice bath with $\mathrm{NaCl}$ at $0{ }^{\circ} \mathrm{C}$ and (ii) using a mineral oil bath at $80{ }^{0} \mathrm{C}$. The variations in experimental conditions used for polymer preparation are presented in Tables 1-3. The bulk polymers were ground in methanol and wet-sieved through $38 \mu \mathrm{m}$ sieves (Endecotts, UK) and sedimented in methanol to remove fines. The polymers were additionally washed out with chloroform containing $0.05 \%$ hexamethylenediamine. Spectrophotometric analysis of ephedrine concentration in washing solutions, performed at $260 \mathrm{~nm}$ indicated that $94-95 \%$ of the template was removed successfully from the polymer. Polymer particles were collected, dried under vacuum and used for packing HPLC columns.

HPLC analysis. For the analysis of MIP recognition properties the polymer particles were suspended in methanol and packed in stainless steel HPLC columns 
(150x4.6mm) at 1000 bar pressure using 1666 HPLC column Slurry Packer (Alltech, UK). The evaluation experiments were carried out using an HPLC system, which included a ConstaMetric-3200 solvent delivery system (LDC Analytical, UK), PerkinElmer ISS-100 automatic injection system and a Waters Lambda-Max Model 481 LC Detector (UK). The separation was performed at ambient temperature. Columns were washed with $0.05 \%$ hexamethylenediamine in chloroform at a constant flow $\left(1 \mathrm{ml} \mathrm{min}^{-1}\right)$ until a stable baseline was achieved. HPLC analysis was performed at a flow-rate of $1.0 \mathrm{ml} \mathrm{min} \mathrm{m}^{-1}$ and monitored by $\mathrm{UV}$ detector at $260 \mathrm{~nm}$. Injection amounts were $8 \mu \mathrm{g}(48.5 \mathrm{nmol})$ in $40 \mu \mathrm{l}$ injection volume. All reported chromatographic data represent the results of $3-5$ concordant experiments. The standard deviation of the measurements was below $5 \%$.

Capacity and separation factors. Capacity factors $\left(\mathrm{K}^{\prime}\right)$ were determined from $\mathrm{K}^{\prime}=$ $\left(t-t_{0}\right) / t_{0}$, where $t$ is the retention time of a given species and $t_{o}$ is the retention time of the void marker (acetone). Effective enantioseparation factors $(\alpha)$ were calculated from the relationship: $\alpha=K^{\prime}(+) / K^{\prime}(-)$, where $K^{\prime}(+)$ and $K^{\prime}(-)$ are the capacity factors of the (+)- and (-)-ephedrine, respectively.

Swelling analysis. Swelling experiments were performed as described previously. Polymer particles (300 mg) with a mesh size of 38-67 $\mu \mathrm{m}$ were packed in $1 \mathrm{ml}$ solidphase extraction cartridges (Supelco, UK). The cartridges were then filled with $1 \mathrm{ml}$ of chloroform. After 6 hours equilibration at $20{ }^{\circ} \mathrm{C}$, the excess solvent was removed from the polymer by applying reduced pressure for 1 minute and the weight of the swollen polymer was measured. The swelling ratio (Sr) of the polymers was calculated from the following equation:

$\mathrm{Sr}=\mathrm{v}_{\mathrm{S}} / \mathrm{v}_{\mathrm{O}}$

Where $\mathrm{v}_{\mathrm{s}}$ is the volume of the swollen polymer and $\mathrm{v}_{\mathrm{o}}$ is the volume of dry polymer. 
Porosity and surface analysis. The determinations of specific surface area were performed using a NOVA "e" surface area and pore size analyser (Quantachrome) based on the nitrogen BET. The adsorption and desorption of nitrogen was used to measure the specific surface area of materials and also the pore size and distribution. Before gas adsorption, the powdered polymer sample (300 mg) was degassed and dried in a vacuum at $100{ }^{\circ} \mathrm{C}$ for 2 hours. 


\section{Captures to Figures.}

Figure 1. Mechanism of decomposition for ACC. ${ }^{12}$

Figure 2. Mechanism of decomposition of the DMPA. ${ }^{15}$

Figure3. Influence of the polymerisation time and ACC percentage on separation factor $(\alpha)$ for polymers synthesized in the oil bath at $80{ }^{\circ} \mathrm{C}$.

Figure 4. Influence of the polymerisation time and ACC percentage on separation factor $(\alpha)$ for polymers synthesized under the UV source.

Figure 5. Influence of the polymerisation time and DMPA percentage on separation factor $(\alpha)$ for polymers synthesized under the UV source.

Figure 6. The temperature profile of the polymerisation reaction with different percentages of initiator for the polymers synthesized in the oil bath at $80{ }^{\circ} \mathrm{C}$.

Figure 7 . The temperature profile of the polymerisation reactions with different percentages of ACC for the polymers synthesized under the UV source.

Figure 8. The temperature profile of the polymerisation reactions with different percentages of DMPA for the polymers synthesized under the UV source.

Figure 9. Influence of the polymerisation time and ACC percentage on swelling ratio for polymers synthesized under the UV source. 
(1)Spivak, D. A.; Shea, K. J. Analytica Chimica Acta 2001, 435, 65-74.

(2)Sellergren, B.; Shea, K. J. Journal of Chromatography 1993, 635, 31-49.

(3) Lanza, F.; Hall, A. J.; Sellergren, B.; Bereczki, A.; Horvai, G.; Bayoudh, S.; Cormack, P. A. G.; Sherrington, D. C. Analytica Chimica Acta 2001, 435, 91106.

(4) Navarro-Villoslada, F.; San Vicente B.; Moreno-Bondi M.C. Analytica Chimica Acta 2004, 504, 149-162.

(5) O'Shannessy, D. J.; Ekberg, B.; Mosbach, K. Analytical Biochemistry 1989, 177, 144-149.

(6) Piletsky, S. A.; Piletska, E. V.; Karim, K.; Freebairn, K. W.; Legge, C. H.; Turner, A. P. F. Macromolecules 2002, 35, 7499-7504.

(7) Sellergren, B.; Dauwe, C.; Scheider, T. Macromolecules 1997, 30, 2454-2459.

(8) Piletsky, S. A.; Guerreiro, A.; Pileska, E. V.; Chianella, I.; Karim, K.; Turner, A. P. F. Macromolecules 2004, 37, 5018-5022.

(9) Haginaka, J.; Sanbe, H. Journal of Chromatography A 2001, 913, 141-146.

(10) Allender, C. J.; Heard, C. M.; Brain, K. R. Chirality 1997, 9, 238-242.

(11) Piletsky, S. A.; Mijangos, I.; Guerreiro, A.; Piletska, E. V.; Chianella, I.; Karim, K.; Turner, A. P. F. Macromolecules 2005, 38, 1410-1414.

(12) Carraher, C. E. Polymer Chemistry; $5^{\text {th }}$ ed. ;Marcel Dekker: New York, 2000.

(13) Xia, W. Z.; Cook, W. D. Polymer 2003, 44, 79-88.

(14) Moad, G.; Solomon, D. H. The chemistry of free radical polymerisation; Pergamon: Oxford, 1995.

(15) Kurdikar, D. L.; Peppas, N. A. Macromolecules 1994, 27, 733-738. 
(16) Goodner, M. D.; Bowman, C. N. Chemical Engineering Science 2002, 57, 887900.

(17) Gruber, H. F. Progress in polymer Science 1992, 17, 953-1044.

\section{Acknowledgements}

This work was supported by EU (IST-2001-33326).

SP would like to acknowledge with gratitude receipt of a Royal Society-Wolfson Research Merit Award.

Financial support from Fundação para a Ciência e a Tecnologia is gratefully acknowledged by AG. 